\title{
Protein Sieving with Capillary Nanogel Electrophoresis
}

Cassandra L. Crihfield, ${ }^{1}$ and Lisa A. Holland ${ }^{1 *}$

${ }^{1}$ C. Eugene Bennett Department of Chemistry, West Virginia University, Morgantown, West Virginia 26506

${ }^{*}$ Author to whom correspondence should be addressed

\begin{abstract}
This supporting information contains a summary of the impact of nanogel concentration on migration times from Figure 1 data, the results of the linear regression analyses associated with Figure 2, plots of retardation coefficients vs protein size, external calibration curves used for quantifying proteins in human serum, impact of hydroxyethyl cellulose on separation performance and linear velocities at nanogel concentrations ranging from $0-30 \%$ and $1.4 \%$ hydroxyethyl cellulose are provided.
\end{abstract}

\section{Table of Contents}

Page S-1, Table S1. Migration time observed at nanogel concentrations of $0,10,20,25$, $30 \%$

Page S-2, Figure S1. Impact of HEC on separation.

Page S-3, Table S2. Linear velocity observed at nanogel concentrations of $0,10,20$, $25,30 \%$ and $1.4 \%$ HEC

Page S-4, Figure S2. Results of linear regression analysis of data obtained for each protein shown in the plot of logarithm of mobility versus nanogel concentration.

Page S-5, Figure S3. Plot of logarithm of relative mobility versus nanogel percentage and linear regression analysis for $20,25,30 \%$ nanogel

Page S-6, Figure S4. Plot of retardation versus protein molecular size for 20,25 , and $30 \%$ nanogel

Page S-7, Figure S5 Electropherograms of serum sample

Page S-8, Figure S6. External calibration curves for quantifying AGP in serum

Page S-9, Figure S7. External calibration curves for quantifying AAT in serum

Page S-10, Figure S8. External calibration curves for quantifying TF in serum 
Table S1. Migration time obtained using different concentrations of nanogel

\begin{tabular}{llllll}
\hline Protein & $\underline{0 \%}$ & $\underline{10 \%}$ & $\underline{20 \%}$ & $\underline{25 \%}$ & $\underline{30 \%}$ \\
trypsin inhibitor & 10.4 & 13.0 & 16.0 & 18.3 & 23.4 \\
$\alpha-1$-antitrypsin & 11.4 & 14.9 & 19.5 & 23.5 & 32.4 \\
transferrin & 17.6 & 23.2 & 29.8 & 35.4 & 50.
\end{tabular}

All migration times had relative standard deviations equal to or less than $1 \%$ with the exception of transferrin at $30 \%$ nanogel, which had a relative standard deviation of $3 \%$. 
Figure S-1

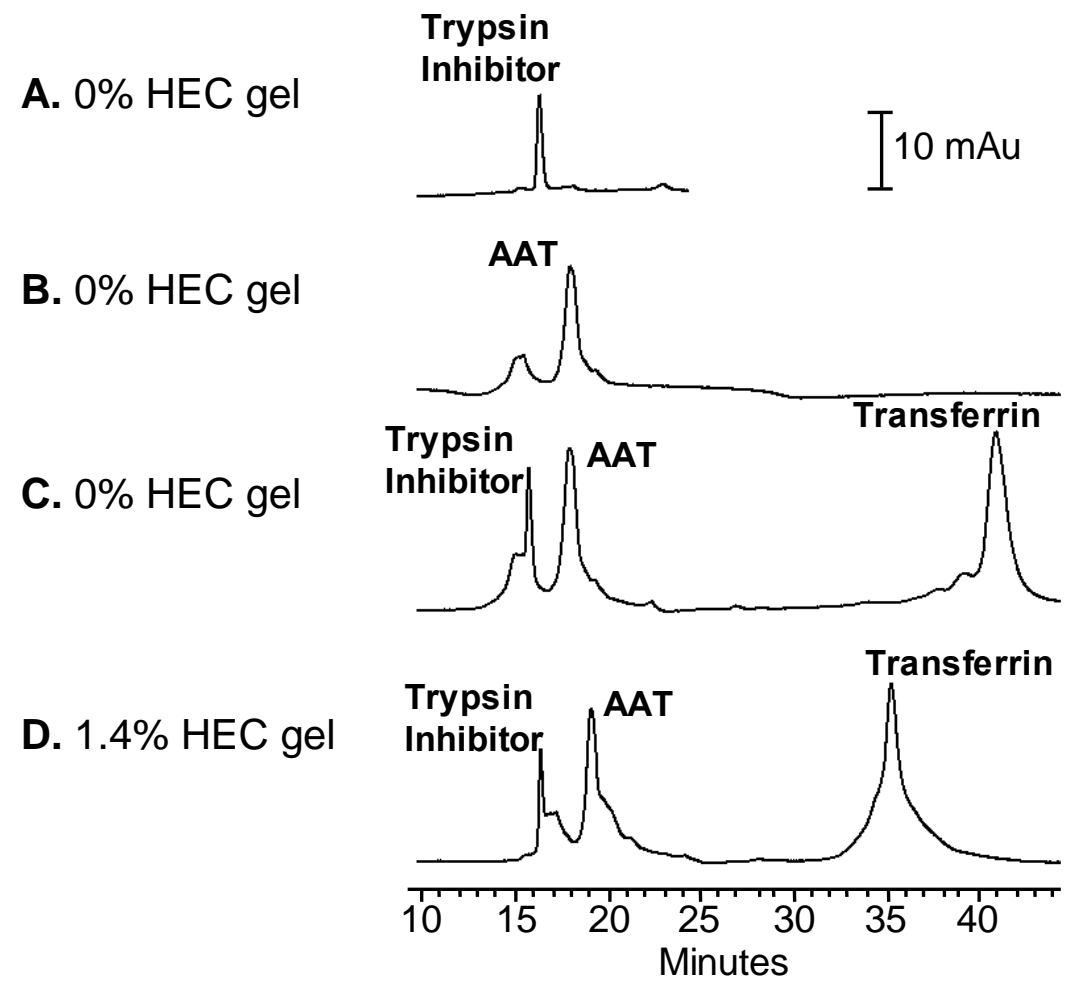

Figure S-1 shows protein separations with a neutral capillary in the absence of gel (S-6A,B,C) and the impact of incorporating 1.4\% hydroxyethylcellulose (HEC) in the capillary (S-6D). The change in migration time of transferrin in the absence (S-6C) and presence (S-6D) of HEC is attributed to the electroosmotic flow in the covalently modified neutral capillary (Sciex 477441), which is directed toward the injection site, in the separation obtained without gel. In the absence of gel (S-6A,B,C) the apparent mobilities for trypsin inhibitor (TI), alpha-1-antitrypsin (AAT) and transferrin (TF) are $1.0 \times 10^{-4} \mathrm{~cm}^{2} / \mathrm{Vs}, 9.0 \times 10^{-5} \mathrm{~cm}^{2} / \mathrm{Vs}$ and $4.0 \times 10^{-5} \mathrm{~cm}^{2} / \mathrm{Vs}$, respectively. The electrophoretic mobility for TI, AAT and TF are $1.0 \times 10^{-4} \mathrm{~cm}^{2} / \mathrm{Vs}, 1.0 \times 10^{-4}$ $\mathrm{cm}^{2} / \mathrm{Vs}$ and $5.0 \times 10^{-5} \mathrm{~cm}^{2} / \mathrm{Vs}$, respectively. The plate count for $\mathrm{TI}$ is $12,000(120,000$ plates $/ \mathrm{m})$, for AAT is 3,000 (30,000 plates $/ \mathrm{m})$ and for TF is $8,000 .(80,000$ plates $/ \mathrm{m})$. In the presence of $1.4 \%$ HEC gel (S-6D) the apparent mobilities for TI, AAT and TF are $1.1 \times 10^{-4}$ $\mathrm{cm}^{2} / \mathrm{Vs}, 8.6 \times 10^{-5} \mathrm{~cm}^{2} / \mathrm{Vs}$ and $4.7 \times 10^{-5} \mathrm{~cm}^{2} / \mathrm{Vs}$, respectively. The electrophoretic mobility for TI, AAT and TF are $1.0 \times 10^{-4} \mathrm{~cm}^{2} / \mathrm{Vs}, 9.7 \times 10^{-5} \mathrm{~cm}^{2} / \mathrm{Vs}$ and $5.7 \times 10^{-5} \mathrm{~cm}^{2} / \mathrm{Vs}$, respectively. In the presence of the gel the plate count for $\mathrm{TI}$ is $18,000(180,000$ plates $/ \mathrm{m})$, for AAT is 5,000 $(50,000$ plates $/ \mathrm{m})$ and for TF is $8,000(80,000$ plates $/ \mathrm{m})$. The resolution for AAT and TI in the absence of HEC (S-A,B,C) is 2.3 compared to 3.4 when HEC is incorporated (S-6D). The resolution for $A A T$ and TF is 15 in the absence of HEC (S-A,B,C) and 12 for neutral coating used with HEC (S-6D). 
Table S2. Linear velocity for nanogels and hydroxyethyl cellulose at $19^{\circ} \mathrm{C}$ and $27^{\circ} \mathrm{C}$

$$
\text { Velocity (cm/min) }
$$

Gel preparation

$\underline{19^{\circ} \mathrm{C}(25 \mathrm{psi})}$

$\underline{27^{\circ} \mathrm{C}(80 \mathrm{psi})}$

Average \pm standard deviation

Average \pm standard deviation

$0 \%$ gel

$$
21 \pm 1
$$

$$
24 \pm 0.6^{*}
$$

$10 \%$ nanogel

$$
18 \pm 2
$$

$0.65 \pm 0.02$

$20 \%$ nanogel

$14 \pm 0.6$

$0.50 \pm 0.02$

$25 \%$ nanogel

$12 \pm 0.8$

$0.44 \pm 0.03$

$30 \%$ nanogel

$11 \pm 2$

$0.40 \pm 0.01$

Gel preparation

$\underline{19^{\circ} \mathrm{C}(80 \mathrm{psi})}$

$\underline{27^{\circ} \mathrm{C}(80 \mathrm{psi})}$

Average \pm standard deviation

Average \pm standard deviation

\section{$1.4 \%$ HEC}

$$
0.51 \pm 0.09
$$

$0.51^{* *}$

Measurements are collected using a $25 \mu \mathrm{m}$ inner diameter fused silica capillary, total length $30 \mathrm{~cm}$, effective length $10 \mathrm{~cm}$. For nanogel, the capillary was filled for 10-minutes at $25 \mathrm{psi}$ with the cartridge temperature set at $19^{\circ} \mathrm{C}$. For hydroxyethylcellulose, the capillary was filled for 20 -minutes at 50 psi with the cartridge temperature set at $19^{\circ} \mathrm{C}$. The instrument was maintained at an ambient temperature of $14-16^{\circ} \mathrm{C}$ with a portable air conditions. Once filled, the separation cartridge was set to either $19^{\circ} \mathrm{C}$ or $27^{\circ} \mathrm{C}$. Once the desired temperature was reached a sample of $1 \mathrm{mM}$ dimethylformamide dissolved in the aqueous BGE was injected (5s, 5 psi for $19^{\circ} \mathrm{C}, 60 \mathrm{~s}, 25 \mathrm{psi}$ for $\left.27^{\circ} \mathrm{C}\right)$ and then pushed to the detection window $(10 \mathrm{~cm})$. The linear velocity is then calculated $(10 \mathrm{~cm} /$ measured time in minutes). At $19^{\circ} \mathrm{C}$ the flow injection analyses were performed using a pressure of $25 \mathrm{psi}$, while at $27^{\circ} \mathrm{C}$ the flow injection analyses were performed using a pressure of 80 psi due to the increased viscosity. All measurements were triplicate, except when noted. The results reported are the average \pm standard deviation.

*This measurement was at 25 psi.

${ }^{* *}$ This velocity is derived from duplicate data $(n=2)$. 


\section{Figure S2}

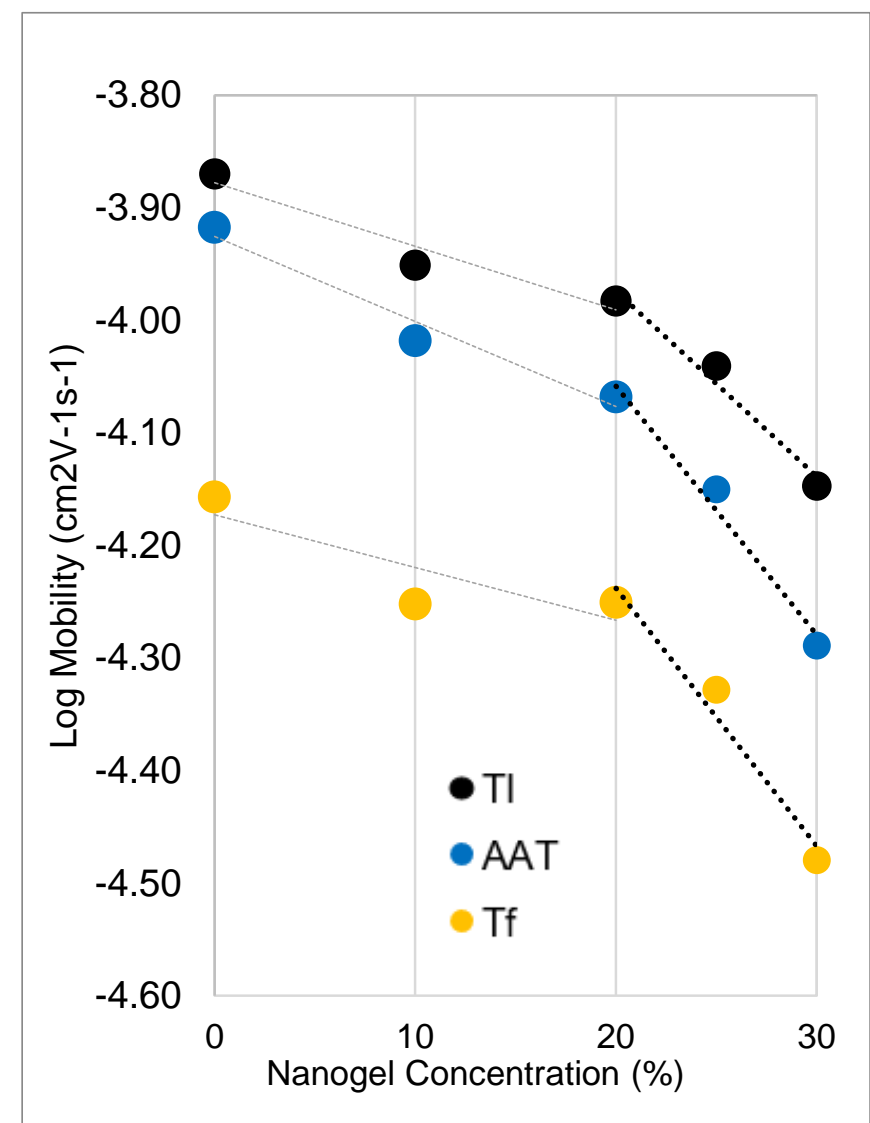

Summary of linear regression of curves in the Figure S-2 plot of the log of mobility versus nanogel concentration ${ }^{\star}$

\begin{tabular}{lccc}
\hline & \multicolumn{3}{c}{$0-20 \%$ nanogel } \\
\hline Protein $^{\text {a }}$ & slope & y-intercept & $\mathbf{R}^{2}$ \\
Trypsin inhibitor & $-0.0056 \pm 0.0008$ & $-3.87 \pm 0.01$ & 0.98 \\
Alpha-1-antitrypsin & $-0.0077 \pm 0.0008$ & $-3.92 \pm 0.01$ & 0.99 \\
Transferrin & $-0.005 \pm 0.002$ & $-4.16 \pm 0.03$ & 0.90
\end{tabular}

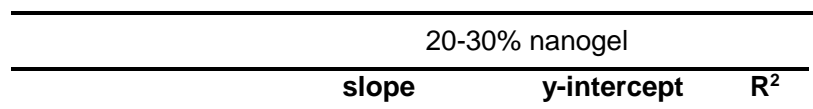

Trypsin inhibitor $\quad-0.016 \pm 0.003 \quad-3.65 \pm 0.07 \quad 0.97$

Alpha-1-antitrypsin $\quad-0.022 \pm 0.003 \quad-3.62 \pm 0.08 \quad 0.98$

$\begin{array}{llll}\text { Transferrin } & -0.023 \pm 0.004 & -3.8 \pm 0.1 & 0.97\end{array}$

${ }^{*}$ Most abundant isoform was used in cases where multiple isoforms were present.

Figure $\mathbf{S 2}$ shows the nanogel concentration versus logarithm mobility plot. The logarithm of the mobility of trypsin inhibitor (TI), alpha-1-antitrypsin (AAT), and transferrin (TF) were plotted versus the percent nanogel. Two distinct slopes were observed for each protein. The beginning of the sieving regime for each protein is indicated by where the two lines intersect. 


\section{Figure S3}

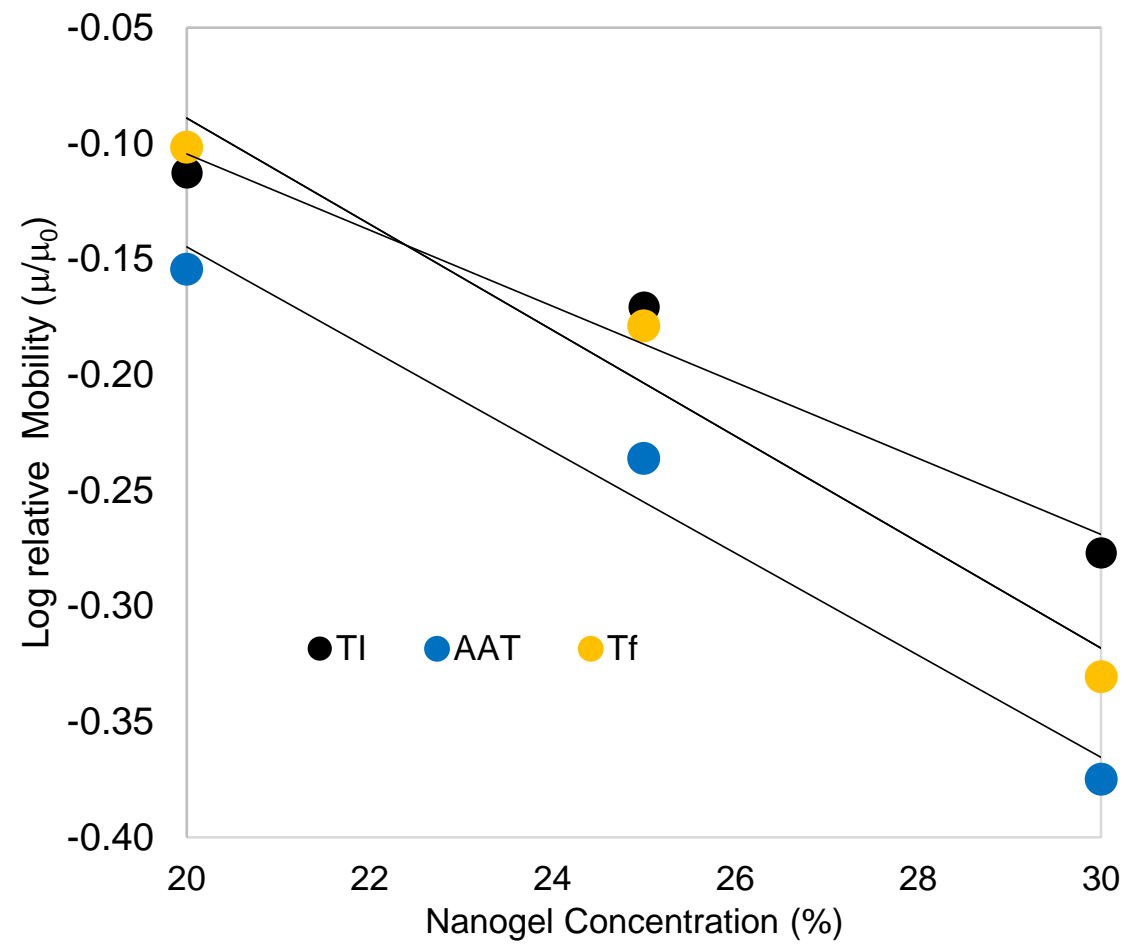

\begin{tabular}{lccc}
\hline $\begin{array}{l}\text { Summary of linear regression of curves in the Figure S-3 plot of the log of } \\
\text { mobility versus nanogel concentration }\end{array}$ \\
\hline \multicolumn{4}{c}{$20-30 \%$ nanogel } \\
\hline $\begin{array}{l}\text { Protein } \\
\text { s }\end{array}$ & y-intercept & $\mathbf{R}^{\mathbf{2}}$ \\
$\begin{array}{l}\text { Trypsin inhibitor } \\
\text { Alpha-1-antitrypsin }\end{array}$ & $-0.016 \pm 0.003$ & $0.224 \pm 0.01$ & 0.98 \\
Transferrin & $-0.022 \pm 0.007$ & $0.30 \pm 0.01$ & 0.99 \\
\hline
\end{tabular}

${ }^{a}$ Most abundant isoform was used in cases where multiple isoforms were present.

Figure S3 shows the nanogel concentration versus logarithm of relative mobility plot. The logarithm of the mobility of trypsin inhibitor ( $\mathrm{TI})$, alpha-1-antitrypsin (AAT), and transferrin (TF) were plotted versus the percent nanogel to obtain the slopes to calculate the retardation coefficient. 
Figure S4

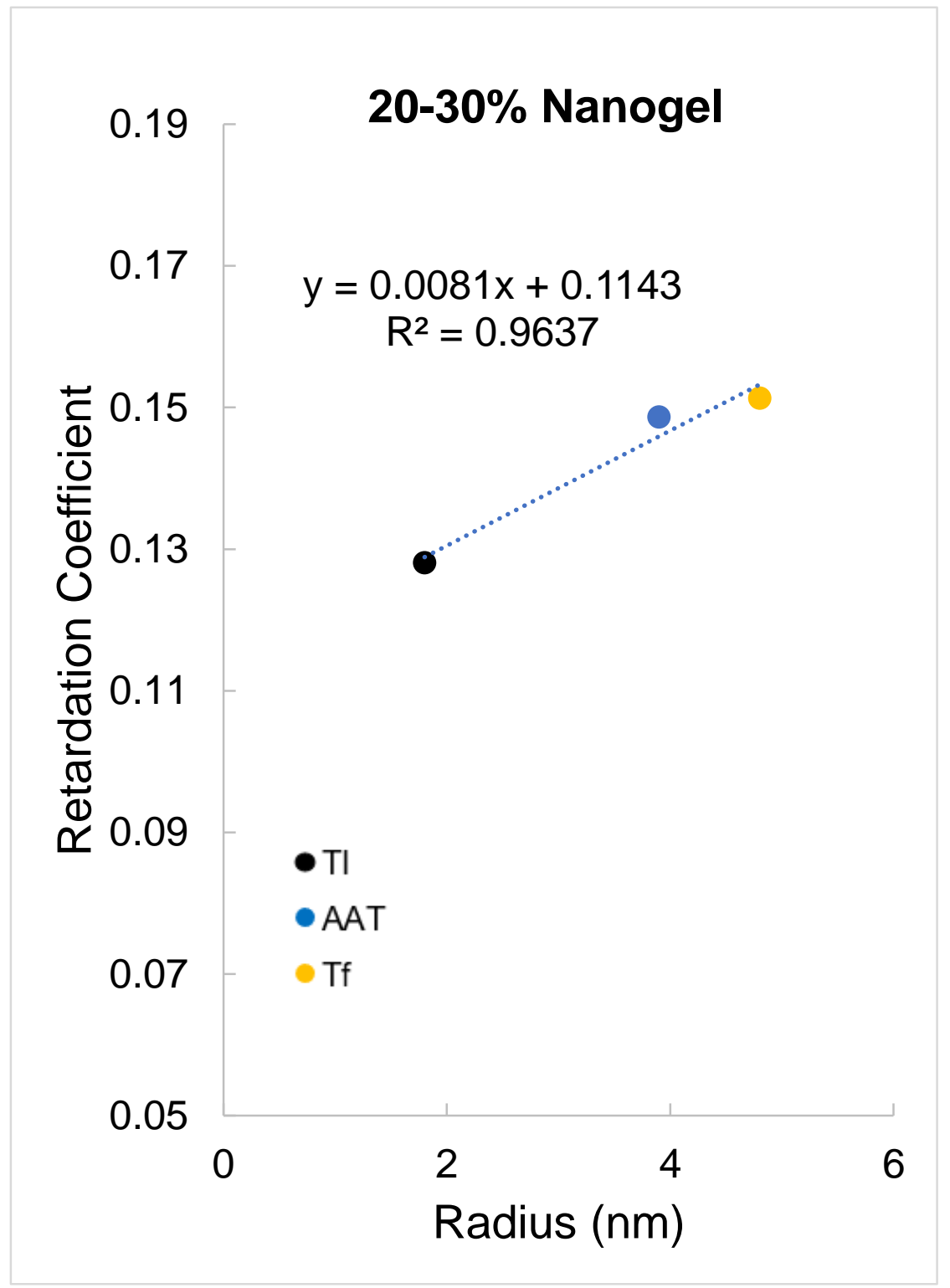

Figure S4 shows the plot of the retardation versus molecular size of trypsin inhibitor, alpha-1-antitrypsin, and transferrin separated using 20-30\% nanogel. 
Figure S5

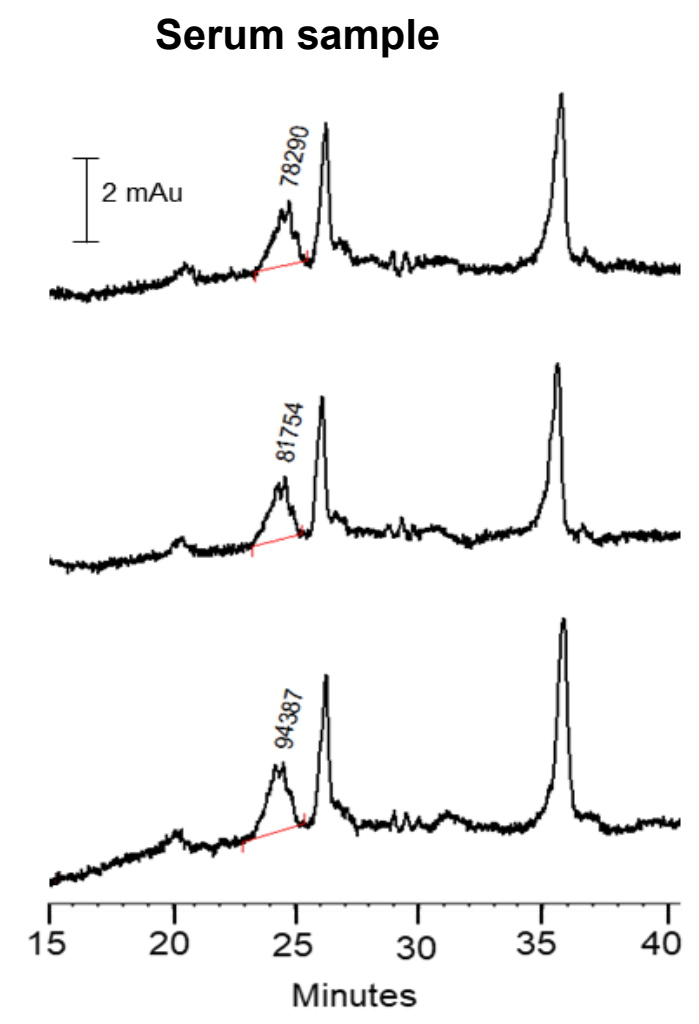

Figure S5 shows the replicate electropherograms of the serum sample. The sample was analyzed using $25 \%$ nanogel and injected at $5 \mathrm{kV}$ for $4 \mathrm{~s}$ injection in order to circumvent low abundance alpha-1-antitrypsin isoforms from contributing to the area of alpha-1-acid glycoprotein. The separations were performed under reversed polarity at $100 \mathrm{~V} / \mathrm{cm}$ at $27^{\circ} \mathrm{C}$. 
Figure S6

A.

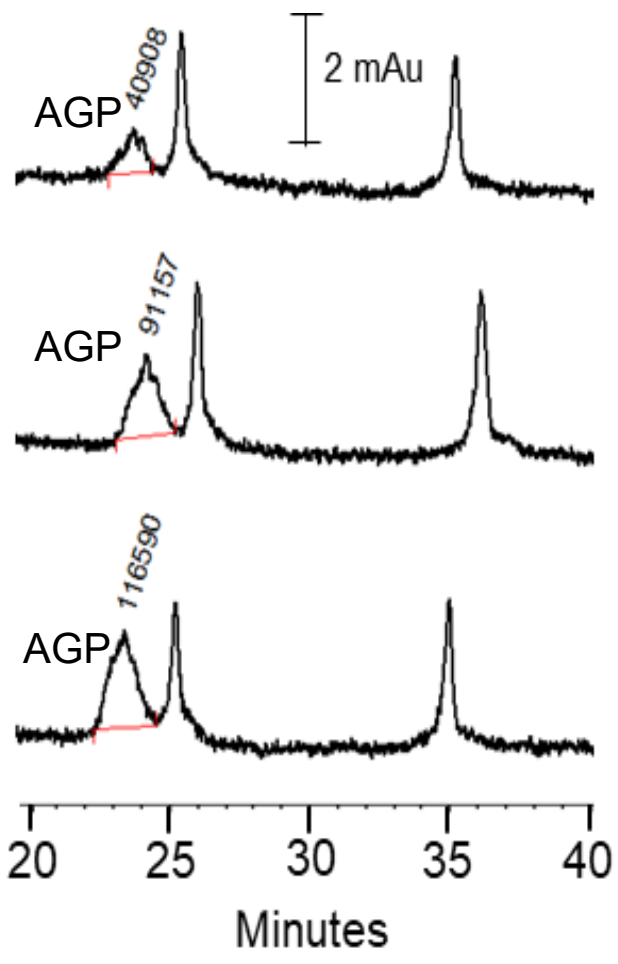

B.

\section{AGP External Calibraiton} Curve

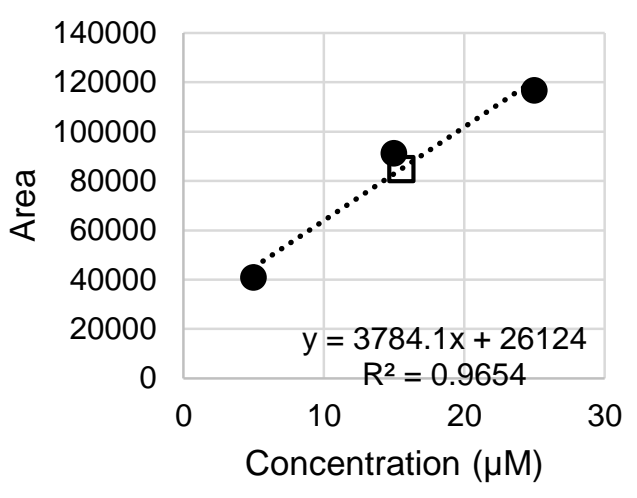

Figure $\mathbf{S} 6$ shows the replicate electropherograms of the alpha-1-acid glycoprotein (AGP) standard (A) used to construct the AGP calibration curve (B). The solid circles $(\bullet)$ are the standard points, while the open squares $(\square)$ represent the serum sample (traces shown in Figure S5). Conditions for the separation are the same as for Figure S5. 


\section{Figure S7}

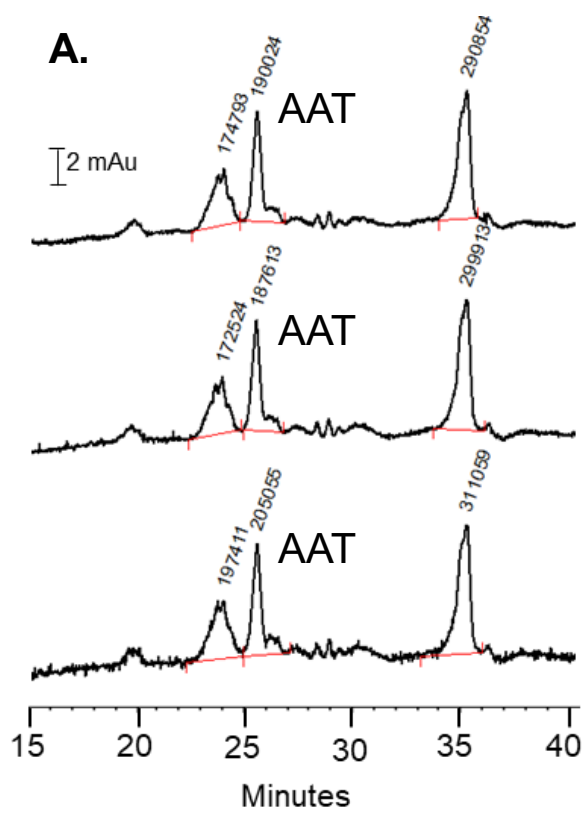

\section{B. AAT External Calibration Curve}

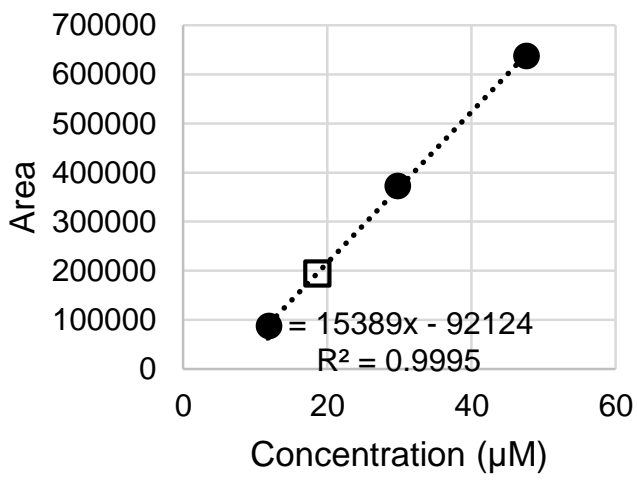

Figure $\mathbf{S} 7$ shows the electropherograms of the alpha-1-antitrypsin (AAT) standards (A) used to make the calibration curve (B). The solid circles $(\bullet)$ are the standard points, while the open squares () represent the serum sample (traces shown in Figure S-5). Injections were performed at $10 \mathrm{kV}$ for $4 \mathrm{~s}$ and all other separation conditions are the same as for Figure S5. 
Figure S8
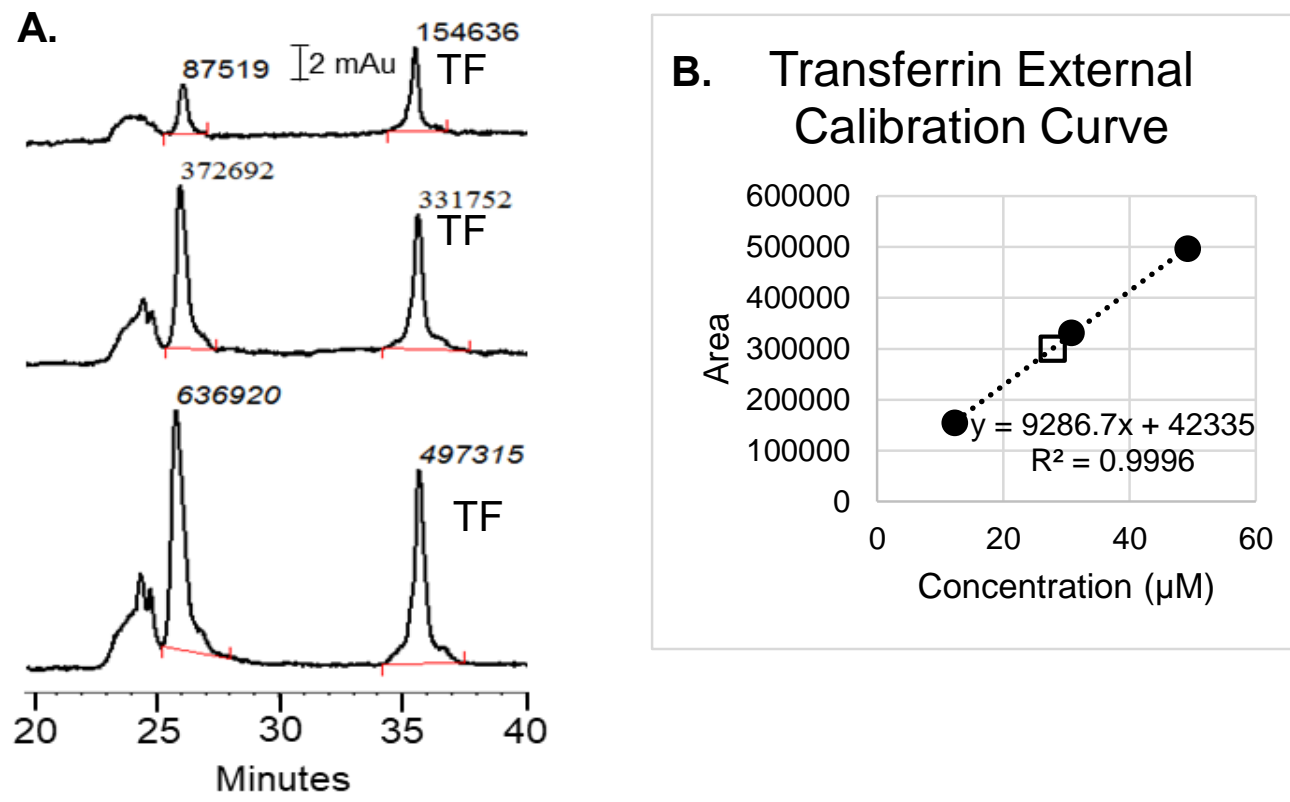

Figure $\mathbf{S} 8$ shows the electropherograms of transferrin (TF) standard (A) used to make the calibration curves (B) quantifying alpha-1-antitrypsin and transferrin in serum. The solid circles $(\bullet)$ are the standard points, while the open squares $(\square)$ represent the serum sample (traces shown in Figure S-5). Injections were performed at $10 \mathrm{kV}$ for $4 \mathrm{~s}$ and all other separation conditions are the same as for Figure S5. 